\title{
Desbuquois Dysplasia
}

National Cancer Institute

\section{Source}

National Cancer Institute. Desbuquois Dysplasia. NCI Thesaurus. Code C124056.

A rare osteochondrodysplasia characterized by short stature, joint laxity, multiple dislocations, vertebral and metaphyseal abnormalities, and advanced carpotarsal ossification. Two forms have been identified: type 1 caused by mutation in the gene CANT1 and type 2 caused by mutations in the gene XYLT1. 